\title{
The ability of using the cavitation phenomenon as a tool to modify the surface characteristics in micro- and in nano-level
} \author{
A. Debeljkovic ${ }^{g}$ \\ a Institute of Nuclear Techniques (INT) of the Budapest University of Technology and Economics (BME), Budapest, Hungary \\ ${ }^{\mathrm{b}}$ Department of Thermohydraulics, Centre for Energy Research, Hungarian Academy of Sciences, Budapest, Hungary \\ ${ }^{\mathrm{c}}$ University of Belgrade, Faculty of Mechanical Engineering, Belgrade, Serbia \\ d Budapest University of Technology and Economics, Department of Electronics Technology, Budapest, Hungary \\ e University of Belgrade, Faculty of Technology and Metallurgy, Belgrade, Serbia \\ ${ }^{\mathrm{f}}$ University of Western Sydney, School of Engineering, Sydney, Australia \\ ${ }^{\mathrm{g}}$ University of Belgrade, Faculty of Mechanical Engineering, NanoLab, Belgrade, Serbia
}

Ezddin Hutli ${ }^{\mathrm{a}, \mathrm{b}, *}$, Milos S. Nedeljkovic ${ }^{\mathrm{c}}$, Attila Bonyár ${ }^{\mathrm{d}}$, Nenad A. Radovic ${ }^{\mathrm{e}}$, Vojislav Llic ${ }^{\mathrm{f}}$,

\section{A R T I C L E I N F O}

\section{Article history:}

Received 20 January 2016

Received in revised form

24 March 2016

Accepted 6 April 2016

Available online 11 April 2016

Keywords:

Cavitation

Nano/micro bubbles

Plastic deformation

Surface Roughness

\begin{abstract}
A B S T R A C T
The aim of this paper is to investigate the possible application of the cavitation phenomenon as efficient method to modify the surface properties (e.g. the surface roughness) in the nano- and micro-levels. Aluminum alloy (AlSiMg) specimens were subjected to high speed submerged cavitating jets under various working conditions, for short time periods between 15 and $30 \mathrm{~s}$. The force generated by the cavitating jet is employed to modify the surface roughness of the specimen. The target surface was analyzed with optical microscopy, white light interferometry, atomic force microscopy (AFM) and also with electrostatic force microscopy (EFM). The results show the possibility to use the cavitation bubbles as a nanofabrication method e.g. for shotless surface peening. With AFM, the deformation mechanism and the formation of planar or wavy slip were also investigated. EFM shows that the changes in the surface roughness also have a strong influence on the electrostatic field above a biased sample.
\end{abstract}

(c) 2016 Elsevier Ltd. All rights reserved.

\section{Introduction}

The phenomenon of cavitation damage is complex since it includes both hydrodynamic and material aspects. Hydro-dynamically, cavitation is characterized by the appearance of vapor bubbles in a liquid subjected to a sudden decrease in pressure below the vapor pressure, in correspondence with the liquid temperature. Collapse of vapor bubbles in the sub-cooled environment creates liquid micro-jets which can cause damage to solid surfaces [1-4].

Cavitation causes different effects that are in general avoided or at least controlled in any hydraulic facility. It is well-known that cavitation can severely damage solid walls by removing material from the surface. On the other hand, cavitation is used in many diverse scientific and industrial applications (jet cutting, underwater cleaning, and improvement of fatigue strength of

\footnotetext{
* Corresponding author at: Institute of Nuclear Techniques, Budapest University of Technology and Economics, Budapest, Hungary. Tel.: (+36-1)-463-2523; fax: (+36-1)-463-1954.

E-mail address: ezddinhutli@yahoo.com (E. Hutli).
}

materials, etc.) with cavitation clouds produced by cavitating jets. It is also well known, that the impingement of cavitating jet leads to serious erosion in valves and associated hydraulic equipment $[1-3,5-7]$.

The cavitation damage starts as a consequence of the contact between the specimen and the liquid microjet, following the collapse of the cavitation bubble (liquid-solid interaction in a microscale). The bubbles themselves are created when the pressure in the liquid falls below its vapor pressure. Collapse is followed by the subsequent vapor pressure increase [8]. The mechanical performance of a material is largely depending on its ability to absorb the shock waves without sustaining microscopic fractures on its surface $[9,10]$.

As well known, the purpose of most laboratory tests regarding the cavitation erosion is to predict the material performance under cavitation attack in a full-scale hydraulic machine or structure. As the course of erosion is generally known to depend essentially on the distribution of the cavitation impacts, the reproduction of this distribution in a laboratory environment may be considered as a condition of reliable quantitative assessments [11]. 
In order to provide a greater understanding of the cavitation damage phenomenon, a number of different testing techniques have been used by researchers to investigate the different cavitation damage stages. Still, this subject is in the focus of investigation, especially, when new technologies are used such as the pit-count technique, the 2D optical method, the 3D measurements by roughness meter, and the 3D laser profilometry technique [12]. Surface roughness is an important factor when dealing with issues such as friction, lubrication, and wear. It also has a major impact on applications involving thermal or electrical resistance, fluid dynamics, noise and vibration control, dimensional tolerance, and abrasive processes, etc. [13]. Surface roughness of metals which are used in the medical field as implants fixed to the bone is an important factor in the recovery process of the patient, because it affects osseointegration. It is reported that increased micro/submicron roughness could enhance the bone cell function [14]. The micro-roughness of the solids plays the main role for the heat transfer in a cross interface, which is important in a wide range of applications, such as microelectronics cooling, spacecraft structures, satellite bolted joints, nuclear engineering, ball bearings, and heat exchangers. The heat transfer mode at the micro-contact is conduction, which leads to a high temperature drop across the interface [15]. Changes in the surface roughness also influence the electrostatic field above a biased sample. In electrostatics the intensity of the electric near field is higher above structures with small geometrical curvature (e.g. sharp features). Such perturbed and intensive near fields could be desirable for some applications [16]. Surface roughness could exert a profound effect on the performance of radio-frequency (RF) cavities or slow wave structures [17-19]. Surface roughness may lead to excessive local electric field enhancement that could trigger RF breakdown [20,21]. Surface roughness may also cause local magnetic field enhancement which can lead to abrupt quenching i.e. loss of superconductivity $[17,22]$.

Considering all points mentioned above surfaces with nano and micro scale roughness with regular or irregular shape, wavy or stripped shape etc. assumed to be important targets for research. Modifying the surface properties with cavitation has several advantages compared to common machining methods used for the same purpose (such as for example abrasive blasting, including shot peening or sandblasting) [42,43]: 1) Compared to shot peening it does not require balls (or other abrasive media besides water), so it is cheaper and cleaner. 2) The cavitation damage can be localized with the jet and the attacked surface area can be very small and/or narrow which can be advantageous for hard to reach areas. 3) The cavitation intensity can be easily controlled by the hydraulic and geometrical parameters. 4) There is no thermal effect to the target surface (compared for example to laser polishing methods). 5) The cavitating jet can be used to selectively pattern the target surface. Such controlled patterning with the jet is possible by selecting an appropriate nozzle (in simpler cases) or by applying a scanning jet and optional thin layers of temporary mask (e.g. a hard coating) to protect local areas.

Therefore the aim of this research is 1) to investigate the effect of cavitation on the surface of the tested AlSiMg alloy during the incubation time period, which refers to the time while only plastic deformation occurs on the surface of the target; and 2) by the characterization of the surface properties prove, that it would be possible to use cavitation as a tool to modify the roughness of surfaces in the sub-micron range in a controlled fashion. White light interferometry and atomic force microscopy (AFM) were used in this work, which - besides the quantitative characterization of surface roughness - can provide additional insight to the deformation process. To investigate the effect of the increased surface roughness on the electrostatic properties of the sample electrostatic force microscopy (EFM) was used.

\section{Facility, cavitation characteristics}

\subsection{Facility setup}

The experimental setup for the investigation of the damage resistance is a closed hydraulic loop. A high-speed submerged cavitating jet was produced in the test chamber by the adjustment of appropriate hydrodynamic conditions and the final outflow to the test chamber through the nozzle. The specimen was prepared and then mounted in the holder in the chamber in front of the nozzle. The chamber was filled with water and then the water was pressurized by a plunger pump. A shortcut line with a pressure gauge functioned as a pressure regulator in the system. The regulation of the water temperature with $\pm 1^{\circ} \mathrm{C}$ precision during the cavitation damage tests was achieved by a cooling circuit with a heat exchanger. The details of the chamber are shown in Fig. 1(a), the details of the test rig are published in a previous work [4].

The cavitating jet impinged on the specimen at $90^{\circ}$ to its surface, as shown in Fig. 1(b). After a certain exposure time the facility was turned off, the chamber was evacuated and the specimen was removed. The specimens were dried, investigated and then the procedure was repeated with other specimens. The intensity of the cavitating jet was controlled via the upstream pressure $\left(P_{1}\right)$ and downstream pressure $\left(P_{2}\right)$, which were measured precisely by transducers and controlled using the needle valves (regulation valves). Filters were employed to remove particles from the circulating water. A temperature regulator and temperature sensors were used to control the water temperature. The nozzle could be mounted in the holder in two ways regarding the inlet and outlet diameters: divergent and/or convergent conicity. The apparatus in the facility (cavitating jet generator) was calibrated in order to obtain results with a high accuracy. The cavitation number was calculated based on the average exit jet velocity assuming one phase flow (the definition of cavitation number proposed by Thema in 1925 is more accurate compared to the ASTM definition, but still not enough). The cavitation number here is assumed to be an average because of the pressure fluctuation, the velocity decays and the compressibility change along the jet coordinates $(R, X)$, thus the cavitation number varies with the points in the jet trajectory on the coordinates $(R, X)[10,23]$. In the experiments the convergent nozzle was used with inlet and outlet diameters of 1 and $0.45 \mathrm{~mm}$ respectively.

\subsection{Cavitation mechanism and characteristics created by cavitating jet generator}

As the high speed liquid jet from the nozzle enters the test chamber filled with liquid, shear stress occurs between the moving and the stagnating liquid as a result of the differences in their velocities. Thus vortices will occur and as a consequence the pressure inside these vortices will decrease to the level of saturation pressure at the working temperature. A number of small spherical bubbles are initially generated in a starting vortex formed at the jet tip and often connected circumferentially with each other in the form similar to a vortex ring (the bubbles are generated when the pressure in the center of the vortex reaches the saturation pressure of the working fluid temperatureconditions of cavitation). The rings of bubbles are connected tighter thus a cone of bubbles is formed around the jet in the shear region (Fig. 2) which decreases the momentum exchange between the jet and the ambient fluid. The effectiveness of the cavitation cone depends on the quantity and radii of the cavitation bubbles. The thickness of the cavitation cone layer is not constant over the full length of the cone (see Fig. 2). The jet is divided into many regions according to the cone state (cavitation state). In the cone development region both the number of bubbles per unit length 
a

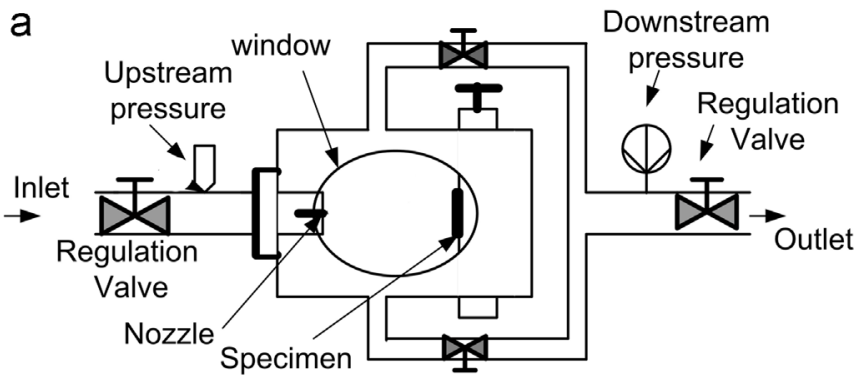

b

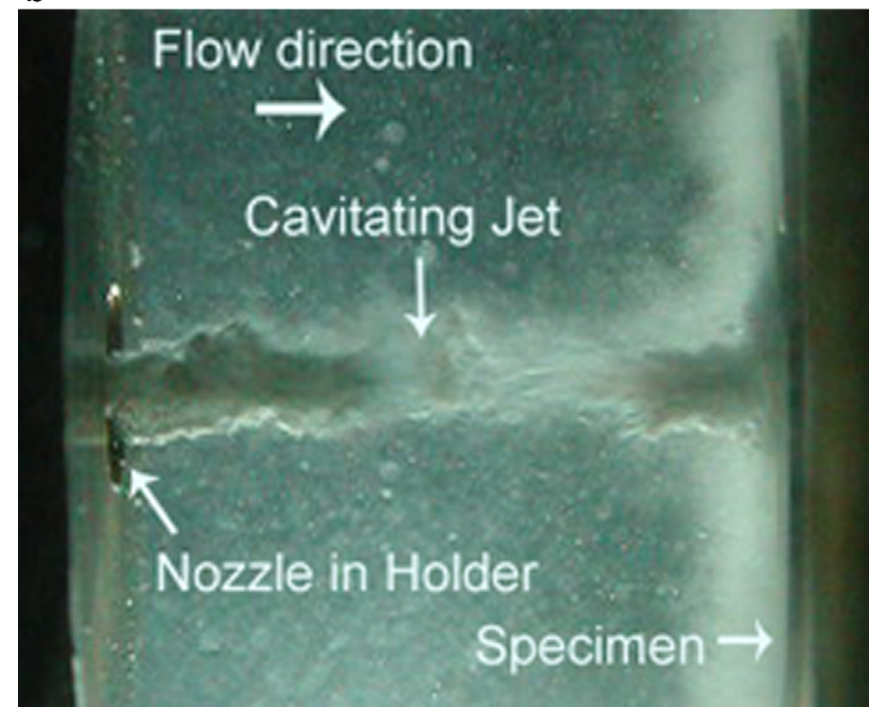

Fig. 1. (a) Schematic diagram of the test chamber. (b) Photo of the cavitating jet colliding with the specimen surface.

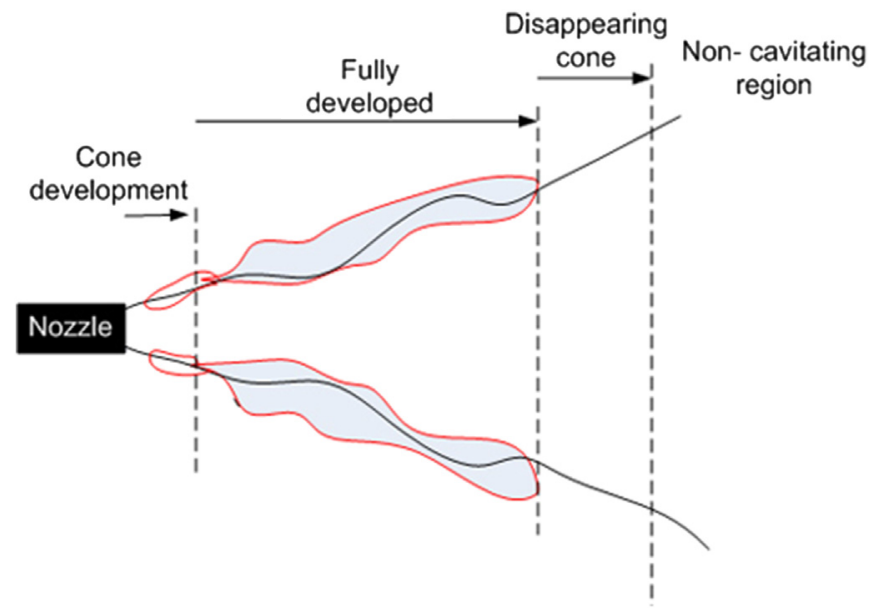

Fig. 2. Cross-sectional scheme of the cavitating jet which represents the various development regions [24].

and their radii increase. After this region the cone is fully developed and the effectiveness is roughly constant (this is the used region in our work). The length of this region depends on the jet pressure, ambient pressure and nozzle diameter. This cone is called cavity cloud, because it contains a huge number of bubbles [24]. In general there are many parameters affecting the bubble dynamics, such as hydrodynamic conditions, working temperature, air content, properties of the working fluid, and geometrical conditions. In this work the main parameters controlling the bubble dynamics are up and downstream pressures, nozzle geometry and working fluid temperature. In fact it is not easy to

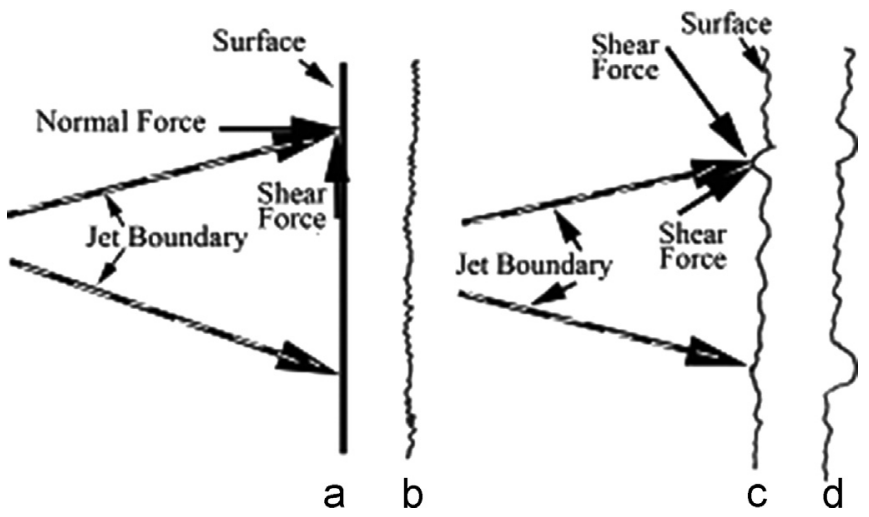

Fig. 3. Sketch of the specimen surface during erosion test: (a) start of the test: contact between the cavitating jet and the smooth specimen; (b) roughened surface after deformation on the surface; (c) contact between cavitaton cloud and roughened specimen; (d) start of rupturing on specimen surface.

control the cavitation bubbles created by the cavitating jet generator because of their huge number, their varying size, their shapes and their contents etc. In a previous publication, the cavitating jet (clouds) dynamics was studied using ordinary, CCD, and high speed photography with framing rates up to 100 thousands frames per second. In addition, the cavity structure composed of bubbles was investigated and their light emission (sono-luminescence) was studied for various temperature and various hydrodynamic conditions. The published results clarified the dynamics of cavitation clouds and highlighted the important parameters related to the control the phenomenon [4,25-27].

\subsection{Proposed model for cavitation-solid interaction}

When a shock wave, emitted in the liquid, impacts a solid wall with an elastic type of behavior, the wall recoils and the shock wave intensity is weakened. As an example, for jets with 2 and $3 \mathrm{~mm}$ in diameter, the decay time from the peak load is 1 or $2 \mu \mathrm{s}$ [33]. At the same time, part of the incident wave is reflected and part is transmitted into the solid.

The plastic properties of metals play an essential role in forming processes and in structural applications. The actual level of stress applied to a metal where elastic deformation turns to plastic deformation is called the proportional limit (the corresponding stress is called yield stress), and is often difficult to determine exactly. The time while the material is under the plastic deformation stage is called the "incubation time", in which plastic deformation occurs on the attacked surface without mass loss. This time is a function of the mechanical properties of the material subjected to this stress (cavitation) when the other parameters (working conditions) are kept constant. The examination of cavitation resistance for a given material should be done in this stage. The plastic deformation can take place only locally; so the ability of the material to absorb impact through local coordination deformation is most important [38]. The accumulation of plastic deformations induces a progressive "work hardening" of the surface layers of the material. Due to this work hardening, the deformation ability of the metals is reduced and the probability of surface cracks and micro-ruptures increases. During this initial stage of cavitation damage, the surface strain increases from zero (for the untreated specimen), up to strains which range between the yield stress (YS=320 MPa) and the ultimate tensile strength (UTS = $370 \mathrm{MPa}$ ) for our investigated AlSiMg alloy. After this stage rupturing of the surface occurs and the process steps to the so called erosion stage.

Fig. 3 shows the possible mechanism (model) for every stages of the cavitation damage (including erosion which was not 
investigated in our experiments). It is assumed that the roughness is increasing with the exposure time during the whole test, enabling the induction of high stresses at the surface, which eventually leads to plastic deformation and rupture at the specimen surface $[34,35]$. The cavitation ring collapses at the moment of its impact on the surface of the specimen. At that very moment, the cavitation ring becomes divided into a large number of small bubbles. The newly formed bubbles vary in size. The interstitial space between them is very narrow, allowing the acceleration of the water to flow between them. This acceleration of liquid leads to an increase in its initial kinetic energy [34,35]. The velocity of the micro/nano-jets is the function of the position of bubble collapsing. In some literature it can be found that, maximum jet velocities are between 50 and $100 \mathrm{~m} / \mathrm{s}$, while others report $950 \mathrm{~m} /$ $s[34,35]$. However, these large deviations between velocities could be reasonable, since the velocity of micro-jets depends on the bubble contents, surrounding pressure and other parameters.

The impact velocity of the micro/nano-jet is much less than the jet velocity (main cavitating jet) because of the momentum and the energy exchange during the jet trajectory. Nevertheless, the jet impact velocity generates a water hammer effect in most cases, and the resulting pressure is often higher than the ultimate tensile strength (UTS) of the target [32,34]. Due to the varying size of the bubbles, the formed "micro-jets" now hit the specimen at angle other than $90^{\circ}$, introducing a shear stress component on the surface. Reported values of the micro-jet induced local material stress are between $100 \mathrm{MPa}$ up to over $1000 \mathrm{MPa}$ [36] (see Fig. 3(a)). The sketch represents the resulting force on the specimen surface (due to oscillations of the cavitation cloud around the theoretical direction and collapsing of cavitation bubbles; these forces are not constant in time, in direction or in intensity). This shear component seems to be sufficient to start plastic deformation on the surface of the specimen, leading to more or less pronounced roughness, as it is shown in Fig. 3(b). After this initial step, further micro-jets hit the roughened surface (instead of the smooth and polished one), Fig. 3(c), which leads to the rupture and erosion of the surface, Fig. 3(d). Following the first observation of surface erosion (which is assumed to be analogous to the crack initiation step) deeper erosion will be triggered within many rings (depending on the depth of the pits). In our current paper we focus on the plastic deformation phase, more details regarding the erosion stage can be found in our previous publication [4].

\subsection{Error analysis of the measured quantities (experimental uncertainty)}

The upstream pressure $\left(P_{1}\right)$ and downstream pressure $\left(P_{2}\right)$ were measured at the inlet and outlet of the test chamber, respectively. The pressure transducers were calibrated by the manufacturer and accuracy certificates were issued for a maximum error of +0.2 / $-0.21 \% \mathrm{FS}$ (full scale, which is 1000 and 10 bar respectively). As the flow rate was determined using $P_{1}$ and $P_{2}$ values from a previous nozzle calibration, its determination uncertainty is also in the order of $\pm 0.3 \% \mathrm{FS}$.

\section{Experimental part}

\subsection{Specimen preparation}

The tested specimens were machined from AlSiMg alloy containing (in wt\%) $0.98 \mathrm{Si}, 0.86 \mathrm{Mn}, 0.64 \mathrm{Mg}, 0.31 \mathrm{Fe}, 0.03 \mathrm{Cu}, 0.08 \mathrm{Zn}$, $0.03 \mathrm{Ti}, 0.02 \mathrm{~Pb}$ and $0.01 \mathrm{Ni}$. The Al-alloy target has a disc shape with $14 \mathrm{~mm}$ in diameter and $2.98 \mathrm{~mm}$ in thickness as can be seen in Fig.3. The full description of experimental setup for cavitation damage testing is given in details elsewhere $[4,25]$. The specimens
Table 1

Hydrodynamic conditions applied in tests with different exposure times.

\begin{tabular}{|c|c|c|c|c|c|}
\hline $\begin{array}{l}\text { Exposure time } \\
\text { (s) }\end{array}$ & $P_{1}$ (bar) & $P_{2}$ (bar) & $V_{j}(\mathrm{~m} / \mathrm{s})$ & $\begin{array}{l}\text { Cavitation number, } \\
\sigma\end{array}$ & $T(\mathrm{~K})$ \\
\hline 15 & $167 \pm 1$ & $3.04 \pm 0.01$ & $121.7 \pm 1$ & $0.0275 \pm 0.001$ & $292 \pm 1$ \\
\hline 20 & $167 \pm 1$ & $3.04 \pm 0.01$ & $121.7 \pm 1$ & $0.0275 \pm 0.001$ & $292 \pm 1$ \\
\hline 30 & $159 \pm 1$ & $3.45 \pm 0.01$ & $118.6 \pm 1$ & $0.029 \pm 0.001$ & $293 \pm 1$ \\
\hline
\end{tabular}

were polished using a classical procedure including grinding papers and diamond pastes. The final paste used was $0.1 \mu \mathrm{m}$. Tests with exposure times between 15 and $30 \mathrm{~s}$ were conducted with the hydrodynamic conditions given in Table 1 . The cavitation number is defined in Eq. (1).

$\sigma=\frac{\left(p_{0}-p_{v}\right)}{0.5 \rho v_{0}^{2}}$

where $p_{v}$ represents the vapor pressure, $p_{0}=p_{2}$ represents the local pressure i.e. downstream pressure, $\rho$ is fluid density and $v_{0}$ represents the exit jet velocity. It is conveniently indicated that, the lower the cavitation number is, the larger is the cavitation risk. The cavitation number $\sigma$ was calculated as shown in the nomenclature. It represents a measure of the resistance of flow to the incidence of cavitation. The value of this parameter was established by the measurements of the upstream and downstream pressures, and by the calculation of the reference velocity from the measured flow-rate.

\subsection{White light interferometry}

To measure the surface properties of the plastically deformed specimens a Zygo New View 7100 white light interferometer was used. Since the whole damaged area is much too large to measure it in one step with the required resolution, multiple images were made on the circular symmetric areas of plastic deformation. Since the extent of this area is changing with the exposure time, the measurement locations were selected with respect to the center of jet impact, as can be seen in Table 2 . The resulting images have a vertical resolution of $0.1 \mathrm{~nm}$, lateral resolution of $300 \mathrm{~nm}$ and scan size of $0.7 \mathrm{~mm} \times 0.94 \mathrm{~mm}$ (10x magnification lens).

Surface roughness $\left(S_{a}, S_{R M S}\right)$ and skewness $\left(S_{s k}\right)$ values are calculated based on the following definitions (Eqs. (2)-(4), where $\eta_{i}$ is the vertical distance from the mean plane to the $z_{i}$ height of a scanned point, $N$ is the total number of points and $\mu_{n}$ is the $n$th central moment calculated for the distribution of height points. Skewness characterizes the symmetry of the height distribution and can yield useful information from rough surfaces e.g. to differentiate between the dominant features (for example: a generally flat surface with some hills on it will have a positive skewness, a flat surface with holes will have negative skewness, while a balanced roughness has small absolute skewness).

$S_{\alpha}=\frac{1}{N} \sum_{i=1}^{N}\left|\eta_{i}\right|$

$$
\begin{aligned}
& S_{q}=\sqrt{\frac{1}{N} \sum_{i=1}^{N} \eta_{i}{ }^{2}=\mu_{2}^{1 / 2}} \\
& S_{s k}=\frac{\frac{1}{N} \sum_{i=1}^{N} \eta_{i}^{3}}{\left(\frac{1}{N} \sum_{i=1}^{N} \eta_{i}{ }^{3}\right)^{3 / 2}}=\frac{\mu_{3}}{\mu_{2}^{3 / 2}}
\end{aligned}
$$


Table 2

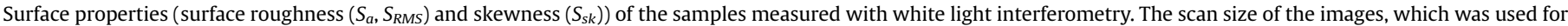
the evaluation was $0.7 \mathrm{~mm} \times 0.94 \mathrm{~mm}$. An average of four-six measurements on different areas are presented, with standard deviations.

\begin{tabular}{|c|c|c|c|c|}
\hline AlMg-alloy & $\begin{array}{l}\text { Measurement position away from the center of jet impact } \\
(\mathrm{mm})\end{array}$ & $S_{a}(\mu \mathrm{m})$ & $S_{R M S}(\mu \mathrm{m})$ & $S_{s k}$ \\
\hline $\begin{array}{l}\text { Reference values, before the cavitation treatment (Exp. } \\
\text { time }=0.0 \mathrm{~s} \text { ) }\end{array}$ & Average of 10 random points on the surface & $0.04(0.01)$ & $0.06(0.03)$ & $-1.29(0.62)$ \\
\hline \multirow[t]{3}{*}{ AlMg-alloy $($ Exp. time $=15 \mathrm{~s})$} & 0 & $0.36(0.05)$ & $0.55(0.03)$ & $-1.66(0.44)$ \\
\hline & 2 & $0.37(0.03)$ & $0.48(0.04)$ & $-1.77(0.52)$ \\
\hline & 4 & $0.12(0.03)$ & $0.23(0.04)$ & $-0.9(0.83)$ \\
\hline \multirow[t]{3}{*}{ AlMg-alloy $($ Exp. time $=20 \mathrm{~s})$} & 0 & $0.43(0.01)$ & $0.64(0.03)$ & $-1.6(0.62)$ \\
\hline & 3 & $1.03(0.07)$ & $1.4(0.13)$ & $-1.28(0.26)$ \\
\hline & 6 & $0.14(0.06)$ & $0.14(0.11)$ & $-3.41(0.81)$ \\
\hline \multirow[t]{4}{*}{ AlMg-alloy (Exp. time $=30 \mathrm{~s}$ ) } & 0 & $0.15(0.08)$ & $0.21(0.13)$ & $-1.37(0.89)$ \\
\hline & 1.5 & $0.47(0.08)$ & $0.64(0.09)$ & $-1.48(0.78)$ \\
\hline & 3 & $0.49(0.11)$ & $0.69(0.13)$ & $-1.36(0.17)$ \\
\hline & 4.5 & $0.14(0.05)$ & $0.23(0.09)$ & -1.79 (0.89) \\
\hline
\end{tabular}

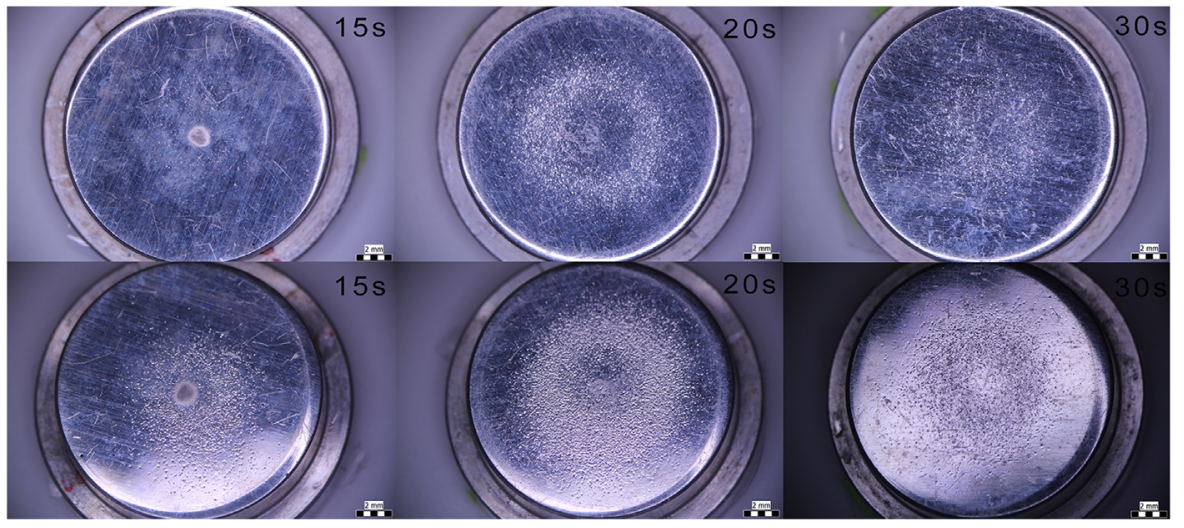

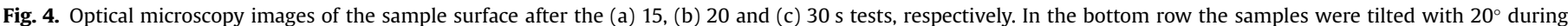
microscopy. The scalebar is $2 \mathrm{~mm}$ in the images.

\subsection{Atomic force microscopy (AFM)}

For the evaluation of the cavitation damage and the characteristic features on the surface of the specimen, atomic force microscopy was used. For this purpose the specimens was carefully removed at regular intervals (exposure times) from the chamber after cavitation attack and dried sufficiently. AFM measurements were done with a JEOL SPM 5200, NanoLab microscope in tapping mode at University of Belgrade, Faculty of Mechanical Engineering. Tapping mode was selected for its ability of high resolution and non-destructive imaging of soft and sensitive samples, which is due to the lack of lateral forces (dragging) between the oscillated tip and the surface $[23,28,29]$. MicoMash NSC36/noAl cantilevers were used, which have $75 \mathrm{kHz}$ nominal resonance frequency, $0.6 \mathrm{~N} / \mathrm{m}$ nominal spring constant and $130 \mu \mathrm{m}$ typical length. The surface roughness was evaluated on images with $60 \mu \mathrm{m} \times 60 \mu \mathrm{m}$ scan size with $256 \times 256$ sampling rate.

\subsection{Electrostatic force microscopy (EFM)}

Electrostatic force microscopy (EFM) measurements were done with a Veeco (lately Bruker) dilnnova type SPM at Department of Electronics Technology, Budapest University of Technology and Economics. The SPM was operated in dynamic lift-off mode with $500 \mathrm{mV}$ bias and $20 \mathrm{~nm}$ lift-off distance between the tip and the sample. Bruker SCM-PIT probes were used, which have $75 \mathrm{kHz}$ nominal resonance frequency and $2.8 \mathrm{~N} / \mathrm{m}$ nominal spring constant. The $100 \mu \mathrm{m} \times 100 \mu \mathrm{m}$ EFM images were recorded with $512 \times 512$ sampling rate and $1 \mathrm{~Hz}$ scan rate. The PID values (the feedback values of the proportional-integral-derivative controller) were optimized according to the user manual. For data evaluation the freeware Gwyddion 2.27 software was used. All AFM and EFM measurements were performed at laboratory ambient temperature and with vibration filtering at both measurement locations $[23,28,29]$.

\section{Results and discussion}

Optical microscopy images of the AlSiMg alloy specimen after the cavitation attack test $(15,20$, and $30 \mathrm{~s}$ respectively) are presented in Fig. 4. The changes on the specimen surface due to the cavitation could be clearly observed on the whole surface, even after this small exposure times. Interestingly, the damage on the specimen is concentrated within a ring, while in the center and on the outer sides of the specimen the damage is much less pronounced. This ring shaped zone of damage on the specimen surface indicates that the bubbles are distributed around the jet in the shear layer zone, while forming a cone shape around the jet [24]. This feature is regulated by the nozzle geometry [4]. The observed smaller cavitation damage in the center of jet impact as well as in the outer fringes of the ring is due to the much lower density of collapsing bubbles, i.e. here the damage process is shifted to much longer times. Others reported similar findings with other techniques (laser and ultrasound generated cavitation) [40,41]. Eroded areas caused by cavitation attack are not visible on the optical microscopy images. The reason for delaying the start of the erosion - considering that the working conditions are constant - is related to the material properties (e.g. elasticity) and the tested "AlMgalloy" has both good ductility and high stacking fault energy. The 

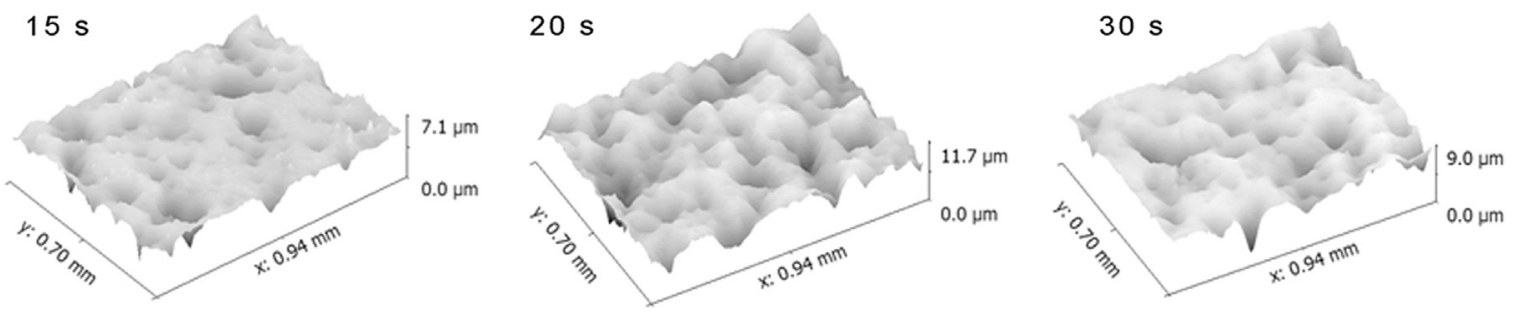

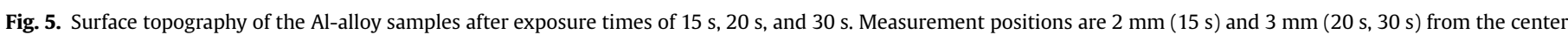
of jet impact.

energy of cavitation caused plastic deformation of the whole specimen, leading to localized modifications on the surface.

Taking into consideration the extent of damage to the sample surface, the quantitative analysis was done in two levels. Since AFM has an insufficient scan range both in the lateral and in the vertical range (the lateral size of the holes can be in the order of $100 \mu \mathrm{m}$, while the maximal scan area of the JEOL SPM 5200 is $60 \mu \mathrm{m} \times 60 \mu \mathrm{m}$ ), white light interferometry was used to obtain surface profiles and calculate the surface roughness of the deformed samples in a statistically reliable way. Due to the circular symmetric nature of the cavitation damage (as can be seen on Fig. 4.) the measurement locations were selected with respect to the center of jet impact. Table 2 collects the quantitative results obtained by the evaluation of four-six $0.7 \mathrm{~mm} \times 0.94 \mathrm{~mm}$ images taken at every specified distance from the impact center (along different axes). Since the area of damage varies with the exposure time, this distance was changed accordingly between the various samples. Fig. 5 presents sample images made at the specified locations at the three sample surfaces.

Based on the obtained surface roughness values presented in Table 2 we can make the following observations: 1) compared to the polished surface (reference), the increase in the surface roughness due to plastic deformation is significant in all of the cases, and it can reach even a $25 x$ increase (after $20 \mathrm{~s}$ ); 2) the circularly symmetric plastic deformation damage is most pronounced in a ring, which is $1.5-3 \mathrm{~mm}$ away from the center of the jet impact (depending on the sample); 3) comparing the $15 \mathrm{~s}$ and 20 s samples - which had the same hydrodynamic conditions - we can say that, by increasing the exposure time both the deformed area and the surface roughness increases with the time; 4) comparing the $30 \mathrm{~s}$ sample with the one treated for $20 \mathrm{~s}$ we can say that due to the smaller jet velocity (and higher cavitation number) both the damaged area and surface roughness is smaller even with this longer exposure time (see Table 1 for details); 5 ) the negative skewness in all of the cases indicates, that the dominant surface features are holes.

In order to have a closer look on the cavitation damage, AFM investigations were done on the treated samples. As a reference for further comparison, Fig. 6 shows three AFM images which present the state of the surface before the tests. The observed straight lines could directly be related to the scratches which were introduced by polishing (the scratches are not deeper than $0.5 \mu \mathrm{m})$.

Fig. 7 shows three sample AFM images obtained on the specimen exposed to the cavitation cloud for $15 \mathrm{~s}$. It can be clearly seen that the roughness of the surface is significantly increased after the treatment, the average for the three images is $\bar{S}_{\alpha}=0.448 \mu \mathrm{m}$. The maximum depths of the pits measured on the three images are $6 \mu \mathrm{m}, 4 \mu \mathrm{m}$ and $1.2 \mu \mathrm{m}$ for Fig. 7(a)-(c) respectively. (Note that due to the $Z$ range limitation of the AFM the total depth of the pit in Fig. 7(a) could not be measured, a plateau can be observed at $-6 \mu \mathrm{m}$.) The total volume of the removed material was estimated around $100 \mu^{3}$ for Fig. 7(a). Although the characteristic deformation of the surface after the treatment is plastic deformation,
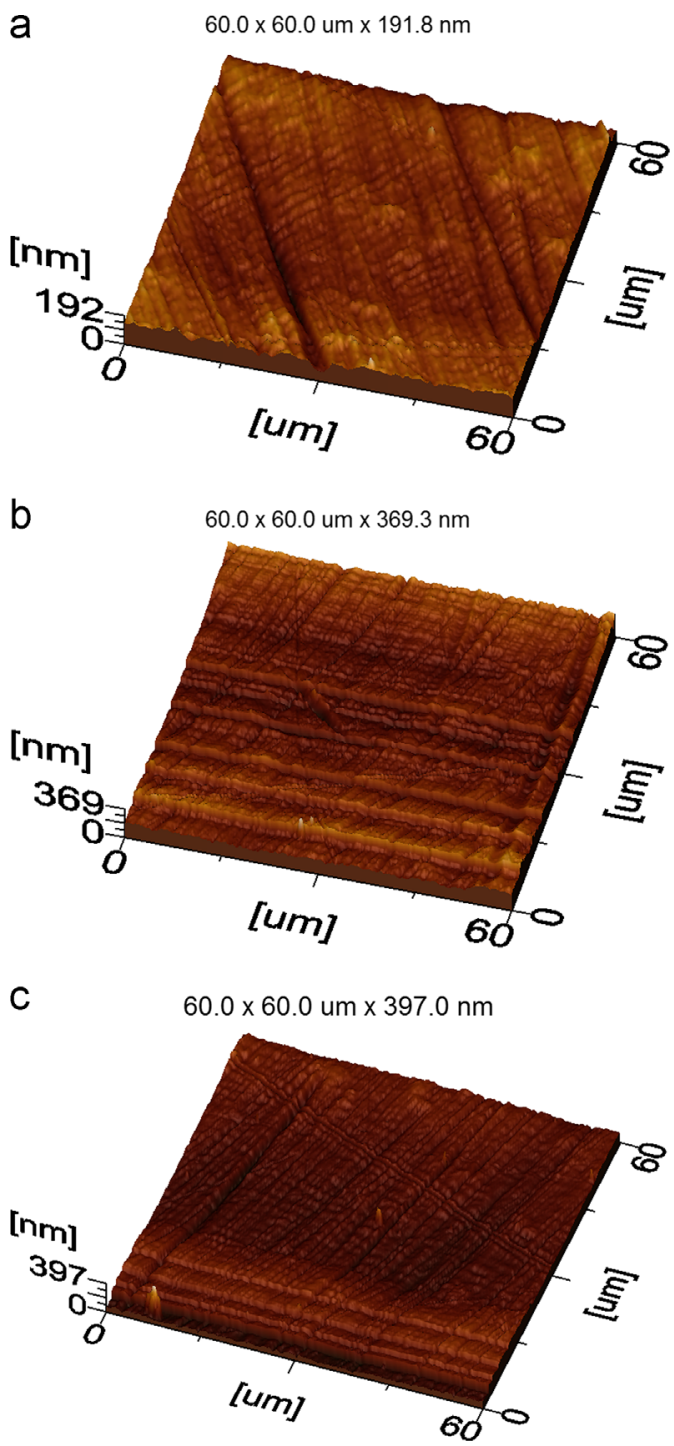

Fig. 6. 3D topography AFM images of the specimen before the test (reference sample) (a) $S_{a}=31.5 \mathrm{~nm}$, (b) $S_{a}=15.4 \mathrm{~nm}$, (c) $S_{a}=34.8$ (average $\bar{S}_{\alpha}=27.23 \mathrm{~nm}$ )

the pit observed in Fig. 7(a) can be attributed to erosion. In this specific location this might be related to some local defect in the material (thus the force of a few bubble collapse was enough to create the erosion), since no erosion could be observed in other areas, not even after longer exposure times. This specific pit proves that erosion can occur - however remotely - during the incubation time period as well if local material defects enable it.

By increasing the exposure time up to $20 \mathrm{~s}$, the damage (plastic-deformation) becomes more pronounced, leading to wider and deeper pits, as it can be seen in the images of Fig. 8. The surface is very irregular and waviness can also be observed. The latter is 
a

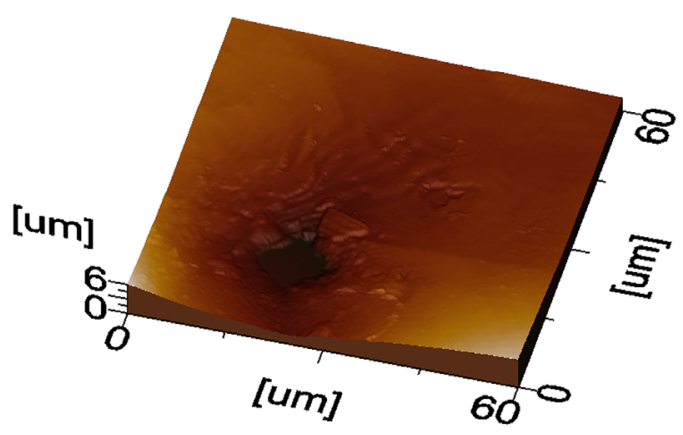

b

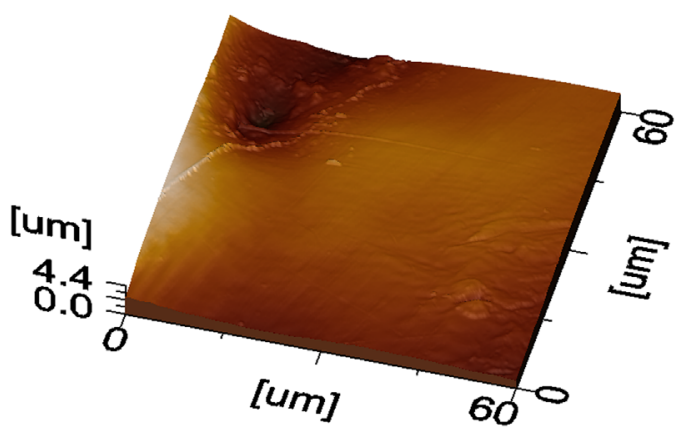

C

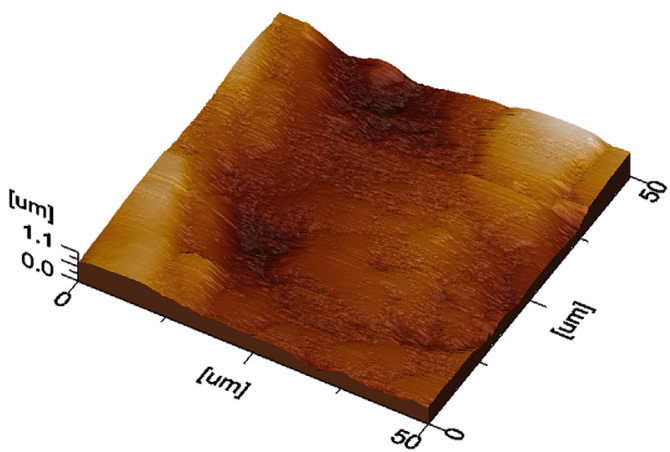

Fig. 7. $3 \mathrm{D}$ topography AFM images of the specimen after the $15 \mathrm{~s}$ test. (a) $S_{a}=0.686 \mu \mathrm{m}$, (b) $S_{a}=0.504 \mu \mathrm{m}$, and (c) $S_{a}=0.153 \mu \mathrm{m} \bar{S}_{\alpha}=0.448 \mu \mathrm{m}$.

even truer for the images of Fig. 9, which shows the surface after $30 \mathrm{~s}$ exposure time.

Based on the presented AFM results several observations can be made. First, it seems that the cavitation bubble collapse has asymmetric features, which correlates well with the previously observed asymmetric shape of the bubbles in the cavitating jet [25], which originates from the highly dynamic, turbulent nature of the jet, where many forces act on the bubbles. Others reported similar asymmetric collapses with laser and ultrasound generated cavitation bubbles as well $[40,41]$. Secondly, the subsequent cavitation bubble rebound and implosion over or near the crater which was produced by the initial collapse is extending the damage. This feature is common in the cavitation damage tests using cavitating jets, it was also reported for other materials e.g. duplex stainless steel [30]. Collective effects also take place when the cloud of cavitating bubbles collapses on the surface following the initial collapse of a single bubble close to a solid wall. Collective collapses are typically characterized by cascades of implosions. The pressure wave emitted by the cavitation bubble collapses as well as a reflection of a particular bubble collapse tends to enhance the collapse velocities of
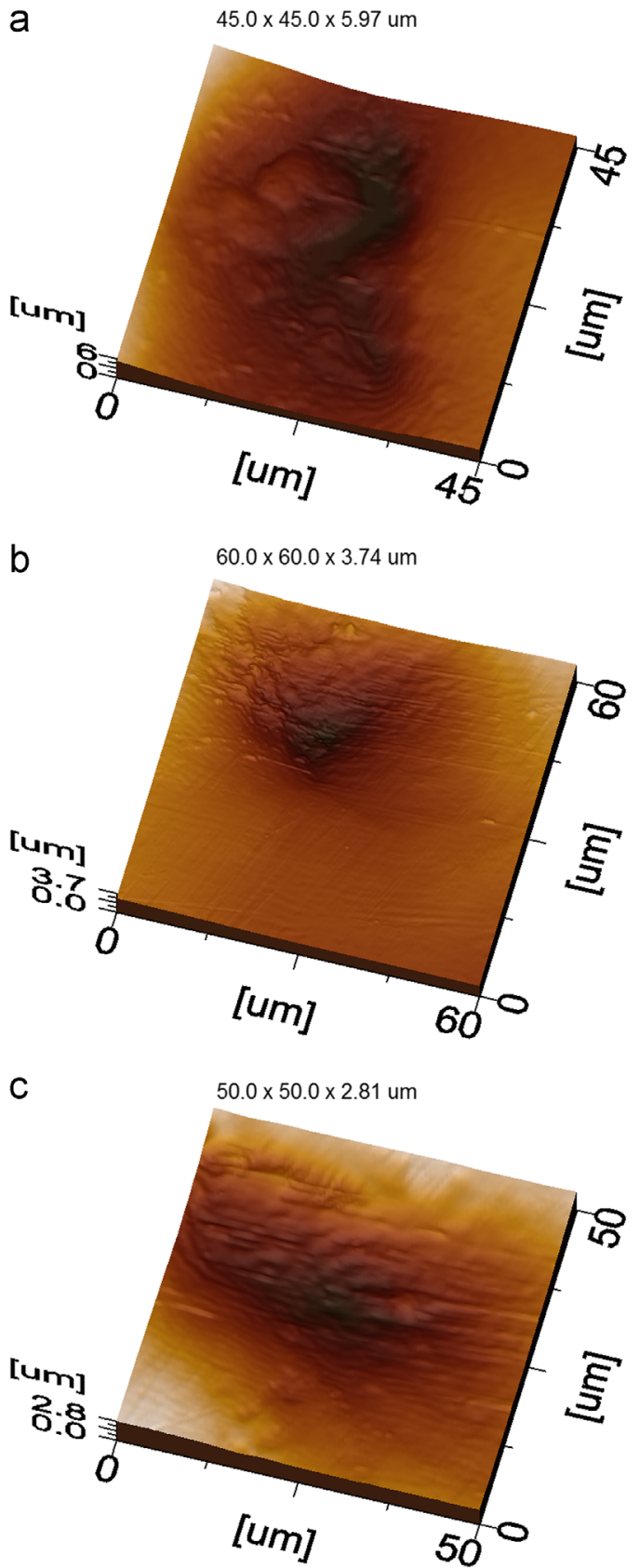

Fig. 8. 3D topography AFM images of the specimen after the $20 \mathrm{~s}$ test. (a) $S_{a}=1.03 \mu \mathrm{m}$ (b) $S_{a}=0.401 \mu \mathrm{m}$, and (c) $S_{a}=0.523 \mu \mathrm{m}, \bar{S}_{\alpha}=0.651 \mu \mathrm{m}$.

the surrounding bubbles, thus increasing the amplitude of their own initial pressure waves [31]. Thirdly, the angle of incidence between the wall surface and the micro/nano-jets is not $90^{\circ}$ in most of the time. The existence of collective collapses indicates the possibility of other angles of incidence. These observations and remarks are in good agreement with the fundamentals of cavitation damage reported previously [31].

During the plastic deformation stage multiple impacts on the same area will result in characteristic features. After the formation of the first pit, further pits can be formed by subsequent impacts which results in deformation bands associated with localized strain, characterized by wavy markings frequently detected on the wall of the pits, as can be seen in Figs. 8(c) and 9(a)-(c). These are the results of a wavy slip or serpentine glide [37], confirming a significant deformation, which can precede a possible rupture (like the one which can be seen in Fig. 7(a)). Further erosion could be 
a

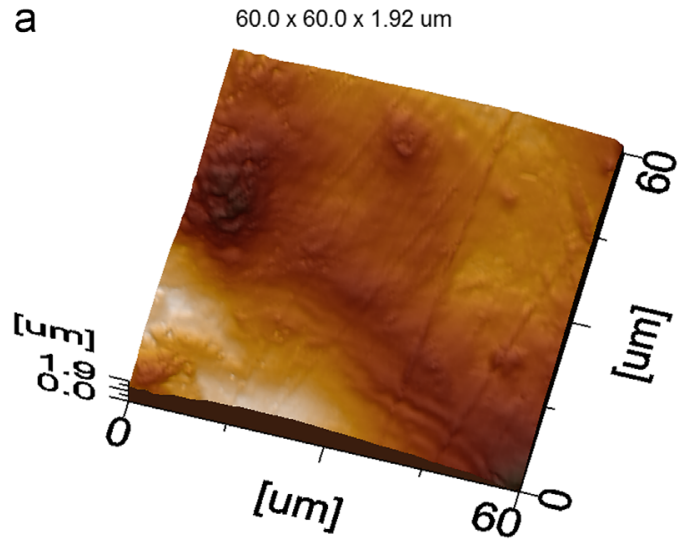

b

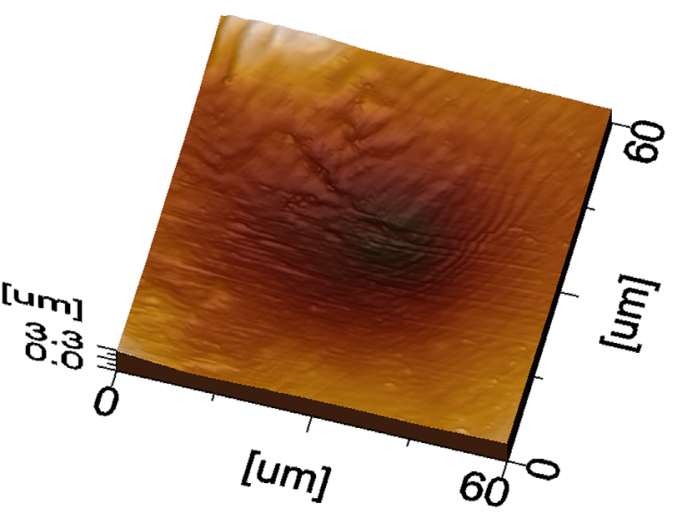

C

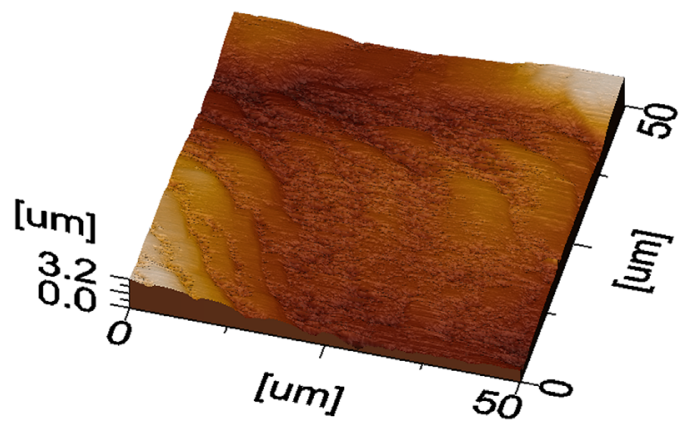

Fig. 9. 3D topography AFM images of the specimen after the $30 \mathrm{~s}$ test, (a) $S_{a}=0.260 \mu \mathrm{m}$ (b) $S_{a}=0.518 \mu \mathrm{m}$, and (c) $S_{a}=0.39 \mu \mathrm{m} ; \bar{S}_{\alpha}=0.389 \mu \mathrm{m}$.

created by the wave-guide model for the acceleration of surface damage $[3,4]$.

The working conditions (cavitation number, exposure time, jet velocity, temperature, etc.), apart from material properties, affect and regulate the behavior of the material during the incubation time period. Note that the working conditions reported here were not constant: in the cases of the tests with 15 and 20 s exposure, the difference between the tests was only in the exposure time, while for the $30 \mathrm{~s}$ exposure test the jet velocity was also changed. For the three test samples the extent of the cavitation damage varied (see Table 2). The most extensive damage (area of damage, depth of pits and surface roughness of the damaged area) was observed at the test with $20 \mathrm{~s}$ exposure time. Therefore, it could be assumed that the jet velocity plays a major role in the resulting surface damage compared to the exposure time. Also, the possible influence of temperature on damage process, which increases during the cavitation bubble collapse, should be taken into account $[3,4]$, this was investigated in our previous publication [4].

Electrostatic force microscopy (EFM) measurements were done on the specimens to investigate the effect of the increased surface roughness on the electrostatic near field of the surface. During EFM an electrically conductive AFM tip - Si or SiN covered with a conductive material such as platinum-iridium - is electrically biased against the sample, while also oscillated in non-contact (or tapping mode) above the surface. In the case of lift-off technique the tip passes two times over each scanned row. During the first pass, the topography is obtained (standard tapping mode), while during the second pass the tip is lifted off to a given fixed distance from the surface, taking into consideration the previously measured topography in that row. During the second pass only the electrostatic forces between the tip and the sample surface modulate the oscillation of the tip. The electrostatic forces $\left(F_{e l}\right)$ between the tip and the surface can be expressed as in Eq. (5), where $V$ is the potential, $C$ is the capacitance of the capacitor formed by the tip and the surface and $z$ is the vertical distance. Note that the potential $V$ contains not only the bias potential, but the differences in the work functions of the applied materials as well. In EFM the electrostatic forces affect the oscillation of the tip, so they modulate the tapping amplitude and phase signals, which are generally used for representation.

$F_{e l}=\frac{1}{2} \frac{\partial C}{\partial z} \Delta V^{2}$

EFM is most commonly used to measure different material properties or embedded charges in the surface. The sharpest contrast in the electrostatic force map can be achieved on surfaces which for example contain conductive and insulating parts, which contain embedded charges, or which has electrically biased parts. However, in the case of materially homogenous and conductive samples, we can presume that the changes in the measured electrostatic map are entirely the effect of the surface roughness on the electrical near field of the surface. When a conductive metallic surface is approached with an electrically charged tip, a mirror charge will be formed on the surface, due to polarization. The interaction between the tip and this 'image' is discussed for example in [39]. In our investigations $500 \mathrm{mV}$ bias potential between the tip and the surface was applied to investigate the electrical near field at $20 \mathrm{~nm}$ above the sample surface.

Fig. 10 presents EFM measurements on the reference sample, before the treatments. The surface roughness is very small $(16.7 \mathrm{~nm})$ and it reflects only the polishing of the surface. The EFM signal (tapping amplitude) correlates well with the topography, but the signal is quite low $(81 \mathrm{mV}$, on a full scale of $20 \mathrm{~V})$.

The electrostatic near field changes significantly in correspondence with the increased surface roughness after cavitation damaging, as can be seen on the EFM images of Fig. 11. The measured surface roughness on the topography image is $112 \mathrm{~nm}$. The changes in the EFM tapping amplitude map are even more drastic, the range of the scale changed to $8 \mathrm{~V}$, which is a 100 fold increase in the signal. This indicates a more intensive interaction between the biased tip and the surface. The measured EFM map correlates well with the topography, so we can presume that the cavitation damage and the created sharp features (holes and hills) locally increase the gradient of the electric field on the surface due to the edge effect. Such intensive near fields could be desirable for some applications [16-22].

\section{Conclusion}

A previously designed testing facility was used to investigate the cavitation damage of surfaces made from an AlSiMn alloy. The 

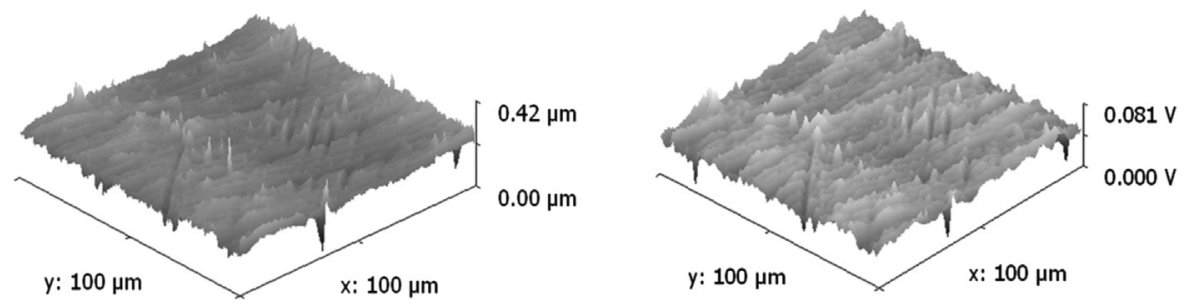

Fig. 10. 3D-EFM images of the reference sample surface before treatment. Left: topography (AFM); right: tapping amplitude (EFM).
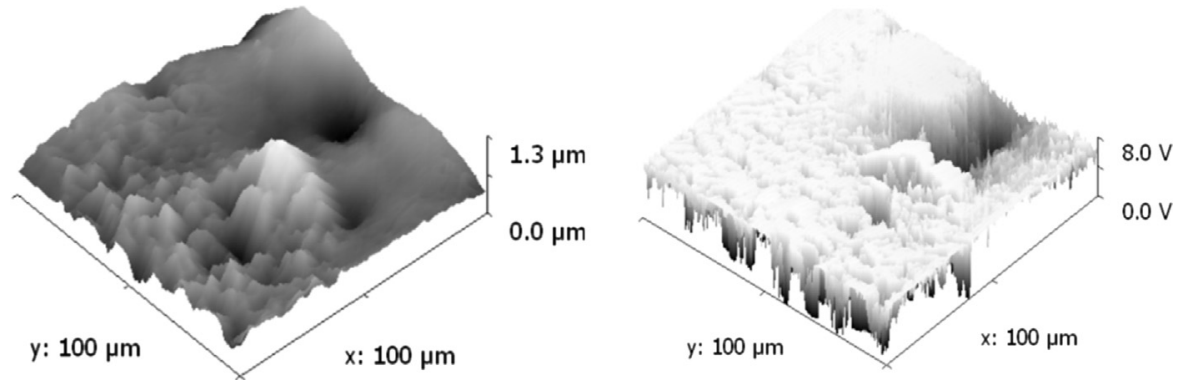

Fig. 11. 3D-EFM images of the sample surface after the $15 \mathrm{~s}$ test. Left: topography (AFM); right: tapping amplitude (EFM).

surface properties of the tested samples were extensively characterized by using white light interferometry, AFM, EFM and optical microscopy. The cavitation damage was present in ring shaped areas, its extent was depending on the working parameters (exposure time, jet velocity, cavitation number) and it indicated the characteristic features of plastic deformation with holes as the dominant structures. We also found that 1) even short exposure times (15-30 s) can increase the surface roughness up to 25-fold compared to the polished reference surface (the measured $S_{a}$ is in the $0.1-1 \mu \mathrm{m}$ range); 2 ) longer exposure times extend the damage and increase the surface roughness; 3 ) jet velocity plays a major role compared to exposure time in terms of damage. Closer AFM investigations on the holes showed signs of wavy slip deformation bands caused by multiple collapses and shear forces acting on the surface. Based on the presented results the cavitation phenomenon seems to be a possible tool to increase the roughness of surfaces in the sub-micron range in a controllable fashion, for desired applications (e.g. shotless peening). EFM shows that the electrostatic characteristic of a biased material changes significantly in correspondence with the increased surface roughness after cavitation damage, therefore cavitation could be also used as tool to modify the electrostatic properties of the materials.

\section{Acknowledgments}

The first author would like to thank the Faculty of Mechanical Engineering, University of Belgrade, Serbia, for the assistance in the AFM measurements, for the Wigner Research Centre for Physics of the Hungarian Academy of Sciences for their help with the interferometry measurements, as well as the Ministry of Science in Libya, for the support through his Ph.D. scholarship. Attila Bonyár is grateful for the support of the János Bolyai Research Scholarship of the Hungarian Academy of Sciences.

\section{References}

[1] Li SC. Cavitation of hydraulic machinery. London: Imperial College Press; 2000. [2] Hammitt FG. Cavitation and multiphase flow phenomena. New York: McGrawHill; 1980.
[3] Knapp RT, Daily JW, Hammit FG. Cavitation. New York: McGraw-Hill; 1970.

[4] Hutli E, Nedeljkovic M, Radovic N. Mechanics of submerged jet cavitating action: material properties, exposure time and temperature effects on erosion. Arch Appl Mech 2008;78:329-41.

[5] Lauterborn WL, Bolle H. Experimental investigations of cavitation-bubble collapse in the neighborhood of a solid boundary. J Fluid Mech 1975;72:391-9.

[6] Dear JP, Field JE. A study of the collapse of arrays of cavities. J Fluid Mech 1988:190:409-25.

[7] Soyama H. Mitsuhiro improvement of fatigue strength of stainless steel by using a cavitating jet with an associated water jet in water. Key Eng Mater 2007:358:162-5.

[8] Qin, Z, Bremhorst K, Alehossein H, Meyer T. Simulation of cavitation bubbles in a convergent-divergent nozzle water jet. J Fluid Mech 2007;573:1-25.

[9] Tomita Y, Shima A. Mechanisms of impulsive pressure generation and damage pit formation by bubble collapse. J Fluid Mech 1986;169:535-64.

[10] Blake JR, Taiba BB, Dohorty G. Transient cavities near solid boundaries, Part 1. Rigid boundary. J Fluid Mech 1986;170:479-97.

[11] Dojcinovic M, Volkov-Husovic T. Cavitation damage of the medium carbon steel: implementation of image analysis. Mater Lett 2008;62:953-6.

[12] Regiane FP, Jean-Luc R, Antoine A. Cavitation damage measurement by 3D laser profilometry. Wear 2000;246:59-67.

[13] Thomas TR. Trends in surface roughness. Int J Mach Tools Manuf 1998;38:405-11.

[14] Jakson MJ, Ahmed W. Surface engineering surgical tools and medical devices. Springer; 2007. p. 21-47 ISBN: 97B-0-387-27026-5http://www.springer.com/ engineering/biomedical + engineering/book/978-0-387-27026-5.

[15] Bahrami M, Culham JR, Yovanovich MM, Schneider GE. Thermal contact resistance of nonconforming rough surfaces, Part 2: thermal model. J Thermophys Heat Transf 2004:18:218-27.

[16] Lazić P, Persson BNJ. Surface-roughness-induced electric-field enhancement and triboluminescence. Europhys Lett 2010. http://dx.doi.org/10.1209/ 0295-5075/91/46003.

[17] John HB. Plasma physics and related challenges of millimeter-wave-to-terahertz and high power microwave generation. Phys Plasmas 2008;15:055502-1-16.

[18] Pandit H, Shi D, Babu NH, Chaud X, Cardwell DA, He P, Isfort D, Tournier R, Mast D, Ferendeci AM. High Tc superconductor re-entrant cavity filter structures. Physica C: Supercond Appl 2005;425:44-51. http://dx.doi.org/10.1016/j. physc.2005.05.010.

[19] Peng Z, Lau YY, Gilgenbach RM. Analysis of radio-frequency absorption and electric and magnetic field enhancements due to surface roughness. J Appl Phys 2009;105:114908-1-1149088.

[20] Caughman JBO, Castano-Giraldo C, Aghazarian M, Baity FW, Rasmussen DA Ruzic DN. Study of RF breakdown mechanisms relevant to an ICH antenna environment. In: Proceedings of the 17th topical AIP conference on radio frequency power in plasmas; 933, 2007, p. 195-202.

[21] Kevin LJ. Electron emission contributions to dark current and its relation to microscopic field enhancement and heating in accelerator structures. Phys Rev Special Top - Accel Beams 2008;11:081001-1-081001-17.

[22] Vasil'ev AA, Gromov AM, Solodukhov GV. Use ofsuperconducting RF systems in low-energy accelerators. Atomic Energy 2005;99:730-4.

[23] Prater CB, Maivald PG, Kjoller KJ, Heaton MG. Tapping mode imaging application and technology. Metrol Instrum Appl Notes 2004 AN04http://www. veeco-europe.com. 
[24] Nobel J, Talmon AM. Measurements of the stagnation pressure in the center of a cavitating jet. Exp Fluids 2012;52:403-15. http://dx.doi.org/10.1007/ s00348-011-1231-y.

[25] Hutli E, Nedeljkovic M. Frequency in shedding/discharging cavitation clouds determined by visualization of a submerged cavitating jet. J Fluids Eng Trans ASME 2008;130:561-8.

[26] Hutli E, Petar B, Nedeljkovic, M. New method to determine shedding/discharging frequency of cavitation clouds based on computer tomography. In: Proceedings of the 15th international conference on fluid flow technologies. Budapest (Hungary); 2008.

[27] Hutli E, Nedeljkovic M, Ilic V. An experimental investigation of cavitating jet dynamic power and cavitation intensity. In: Proceedings of the ASME 2010 international mechanical engineering congress and exposition, 7, Vancouver, British Columbia, Canada, ISBN: 978-0-7918-4444-1; 2010.

[28] Bojovic B, Milkovic ZD, Babic BR, Koruga DLJ. Fractal analysis for biosurface comparison and behavior prediction. Hem. Ind. 2009;63:239-45.

[29] Kojic D, Matija L, Petrov L, Mitrovic R, Koruga D. Surface characterization of $\mathrm{Pb} 1-\mathrm{XMnXTe}$ alloy by atomic force microscopy and magnetic force mode. Surf Eng 2011;27:158-63.

[30] Karimi A. Cavitation erosion of duplex stainless steel. Mater Sci Eng 1987;86:191-5.

[31] Franc JP, Michel JM. Fluid Mechanics and Its Applications ISSN 0926-5112, In: Thess, André (Ed.), Fundamentals of cavitation, chapter 12-cavitation erosion, Springer Netherlands; 76, 2006, p. 265-91.

[32] Chen HS, Wang JD, Li YJ, Chen DR. Effect of hydrodynamic pressures near solid surfaces in the incubation stage of cavitation erosion. Proc Inst Mech Eng Part J: J Eng Tribol 2008;222(4):523-31.
[33] Brunton JH. High speed liquid impact. Philos Trans R Soc Lond Ser A Math Phys Sci 1996:79-85.

[34] Brujan EA, Nahen K, Schmidt P, Vogel A. Dynamics of laser-induced cavitation bubbles near an elastic boundary. J Fluid Mech 2001;433:251-81.

[35] Lauterborn W. Kavitation durch laserlicht. Acustica 1974;31:51-78.

[36] Karimi A, Martin JL. Cavitation erosion of materials. Int Met Rev 1986;31:1-26.

[37] Van Stone RH, Cox TB, Low JR, Psioda JA. Microstructural aspects of fracture by dimpled rupture. Int Met Rev 1985;30:157-79.

[38] Wenge C, Chenqing G, Kang Z, Fusan S. Correlation of cavitation erosion resistance and mechanical properties of some engineering steels. J Mater Sci 2006; $41: 2151-3$.

[39] Kantorovich LN, Livshits AI, Stoneham M. Electrostatic energy calculation for the interpretation of scanning probe microscopy experiments. J Phys: Condens Matter 2000;12:795.

[40] Tzanakis I, Eskin DG, Georgoulas A, Fytanidis DK. Incubation pit analysis and calculation of the hydrodynamic impact pressure from the implosion of an acoustic cavitation bubble. Ultrason Sonochem 2014;21:866-78.

[41] Haosheng C, Jiang L, Darong C, Jiadao W. Damages on steel surface at the incubation stage of the vibration cavitation erosion in water. Wear 2008;265:692-8.

[42] Soyama H. Increase of ability of water jet by using cavitation impacts and its application to peening of material. J Jet Flow Eng 1999;16(3):22-8.

[43] Soyama H, Asahara M, Saka M. Hybrid surface treatment of carbon steel by using a cavitating jet. Trans Eng Sci 1999;25:93-102. 